\title{
Leiomyomas of the lower respiratory tract
}

\author{
SH WHITE, NBN IBRAHIM, CP FORRESTER-WOOD, K JEYASINGHAM \\ From the Departments of Thoracic Surgery and Pathology, Frenchay Hospital, Bristol
}

ABSTRACT Leiomyomas account for about $2 \%$ of benign tumours of the lower respiratory tract. From the relatively few cases reported in the literature, it appears that these tumours most commonly occur in the fourth decade, although one third of patients are under the age of 20 years. The distribution of leiomyomas favours the distal part of the tracheobronchial tree and the most common site for tracheal lesions is the membranous portion of the lower third. Over $90 \%$ of pulmonary parenchymal leiomyomas, which themselves are more common in women, are incidental findings on chest radiographs whereas bronchial lesions are important causes of irreversible lung damage. Tracheal lesions may present as bronchial asthma. Accurate early diagnosis rests on a high index of clinical suspicion and histological examination of bronchoscopic biopsy specimens or frozen section material obtained at exploratory thoracotomy. Treatment could be conservative surgery, but $65 \%$ of reported cases have been managed by lobectomy or pneumonectomy as a result of advanced irreversible lung disease or unawareness of the benign nature of the lesion.

Several individual and collected case reports of leiomyoma of the lung,,$^{2}$ bronchus,,$^{34}$ and trachea ${ }^{56}$ have been published. Such tumours are rare, and so far only 65 cases have been reported. ${ }^{1-13}$ In this article we report three cases of leiomyoma of the lower respiratory tract, which illustrate the three distinct anatomical varieties - namely parenchymal, bronchial, and tracheal leiomyomas.

\section{Case reports}

\section{CASE 1}

A 54 year old man was admitted for investigation in November 1983 . One year previously he had had an episode of bilateral pneumonia and left ventricular failure, for which he was treated at another hospital. He made a complete recovery but follow up chest radiographs showed a persistent right hilar shadow. There was no other important past medical history and his only medication was digoxin. On admission he was mildly obese but there were no abnormal cardiovascular or respiratory symptoms or signs. Lung function studies showed a vital capacity of 3.43 litres and an $\mathrm{FEV}_{1}$ of 2.731 . Conventional

Address for reprint requests: Dr Nassif BN Ibrahim, Department of Histopathology, Frenchay Hospital, Bristol BS16 1LE.

Accepted 1984 anteroposterior tomograms showed a peribronchial opacity in the upper part of the right hilum, best defined at $14 \mathrm{~cm}$ from the plate.

Bronchoscopy showed diffuse inflammation just distal to the bifurcation of the right main bronchus and narrowing at the origin of the right upper lobe bronchus. The intersegmental carinae in the upper lobe bronchus were widened, suggesting an extrabronchial lesion. Bronchial brushing smears showed no malignant cells and bronchial biopsy showed no evidence of tumour.

With a presumptive diagnosis of bronchogenic carcinoma the patient underwent a right exploratory thoracotomy. The lung was found to contain a large rounded mass arising in the parenchyma of the upper lobe at its junction with the hilum (fig 1), which was well defined but adherent to the surrounding structures, including the phrenic nerve, superior vena cava, and azygos vein. When the lesion had been separated from these structures by dissection, a biopsy was obtained and histological examination of a frozen section showed a vascular leiomyoma, the diagnosis being later confirmed by examination of a paraffin section (fig 2 ) and by electron microscopy of the resected lesion (fig 3). The postoperative course was uneventful and he was discharged on the 11 th postoperative day.

CASE 2

A 46 year old man was referred by his medical prac- 


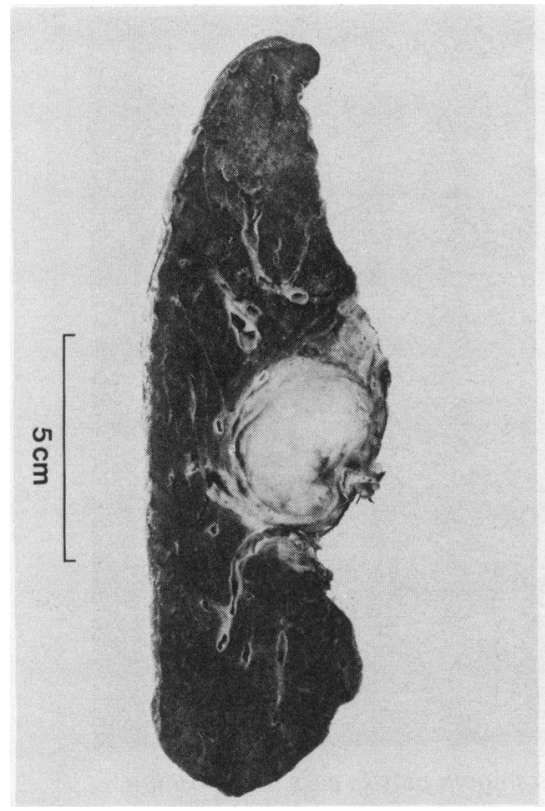

Fig 1 Case 1: Upper and middle lobe of right lung showing a hilar, greyish white, well demarcated tumour, which was slightly furm and showed a whorled pattern in places.

titioner in October 1983 with a one week history of recurrent haemoptysis and weight loss of $10 \mathrm{~kg}$ over two months. In addition, he complained of vague left sided chest pains and general malaise. There was no history of cough, fever, or night sweats. He smoked 10 cigarettes a day and was having no medi- cation. On examination he was a pale, thin man with no respiratory or other physical signs. Posteroanterior and lateral chest radiographs and chest tomograms were normal, but tomography of the upper trachea showed a round intraluminal tumour about $1 \mathrm{~cm}$ in diameter and $10 \mathrm{~cm}$ above the carina (fig 4). Bronchoscopy showed a small sessile nodule about $5 \mathrm{~cm}$ below the vocal cords on the posterior membranous portion of the trachea. The rest of the bronchial tree appeared normal. Brushing smears from the lesion showed no malignant cells and biopsy of the tracheal nodule revealed a benign leiomyoma. The broad base of the nodule ruled out endoscopic resection and the tumour was therefore excised with a small margin of normal membranous wall. The defect was repaired with four absorbable sutures. Histological examination confirmed the diagnosis of a benign leiomyoma. The patient made an uneventful postoperative recovery and was discharged on the seventh postoperative day.

\section{CASE 3}

A 5 year old boy was admitted in August 1956 for investigation of chronic left lung disease. He was healthy with normal milestones until 4 years of age, when he had two episodes of pneumonia within four months, after which he remained breathless on exertion, with a persistent cough. On admission a chest radiograph showed complete collapse of the left lung and gross displacement of the mediastinum to the left. Bronchograms suggested obstruction of the left main bronchus at the level of the carina. Bronchoscopy confirmed complete obstruction of the bronchus with a soft tumour. As much tumour as

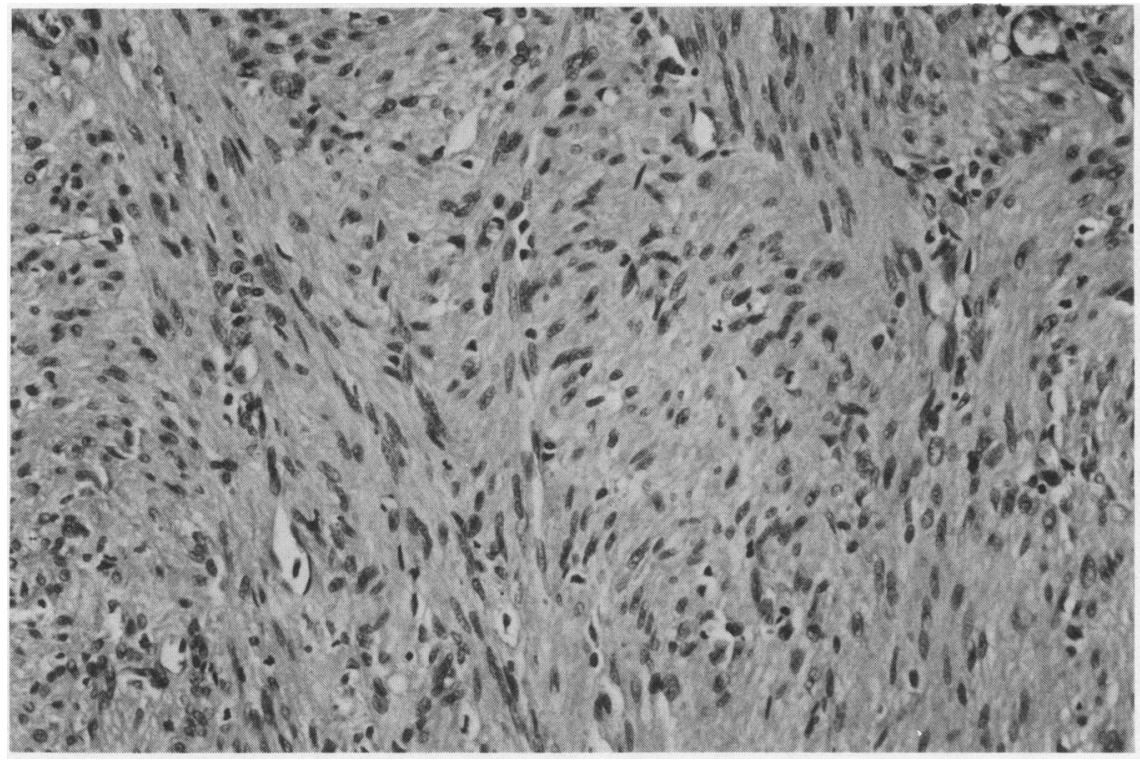

Fig 2 Case 1: Benign vascular leiomyoma, with fibrous component. Note the absence of mitotic figures and the uniformity of the tumour cells. (Haemotoxylin and eosin, $\times$ 180.) 


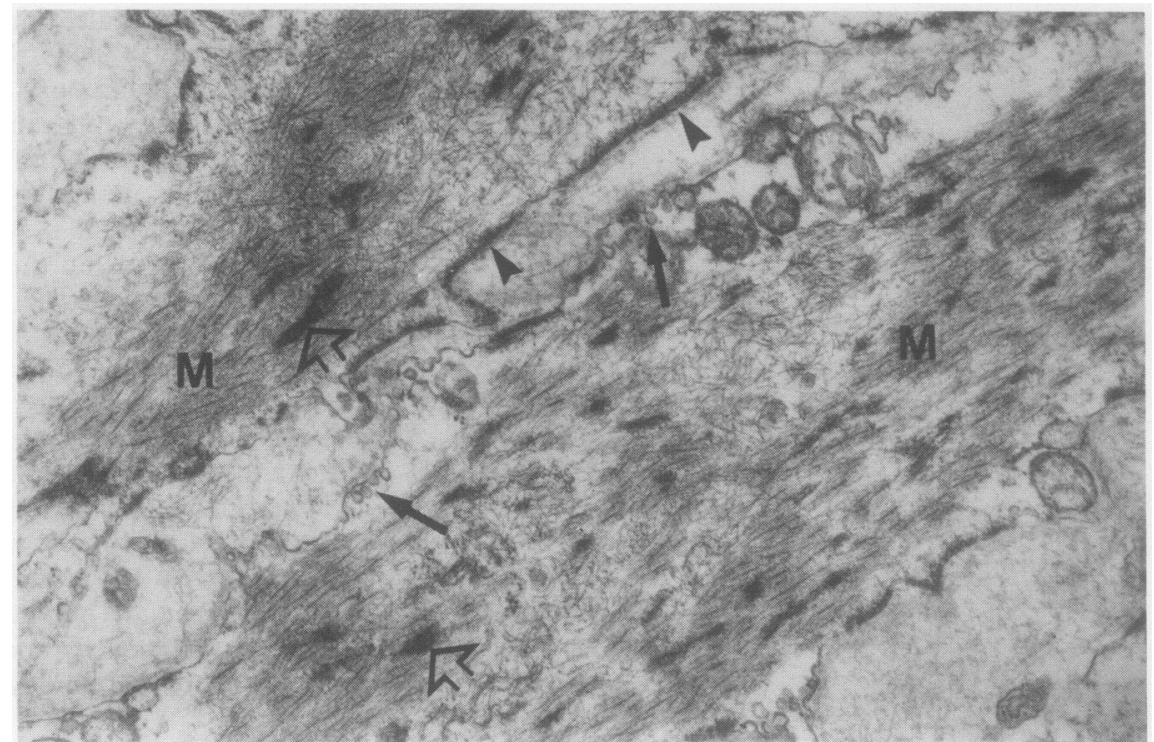

Fig 3 Case 1: Electron micrograph showing parts of two smooth muscle cells in apposition. Many myofilaments $(M)$ with focal densities (outlined arrows), pinocytotic vesicles (long arrows), and dense plaques (arrow heads) are present. (Formalin fixed material, uranyl acetate and lead citrate, $\times 17$ 500.)

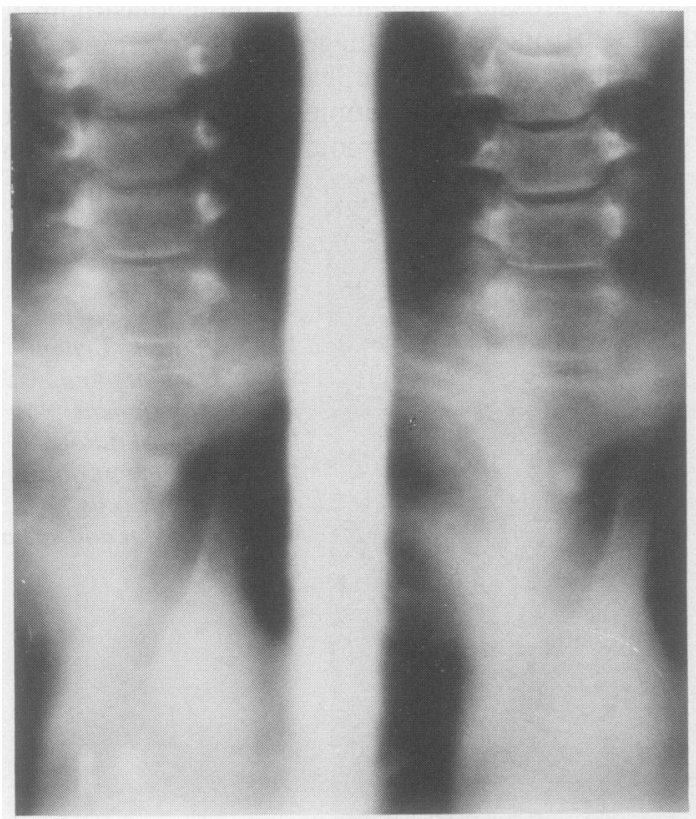

Fig 4 Case 2: Anteroposterior tomogram of tracheal inlet showing a well circumscribed lesion, best seen in the $14 \mathrm{~cm}$ cut.

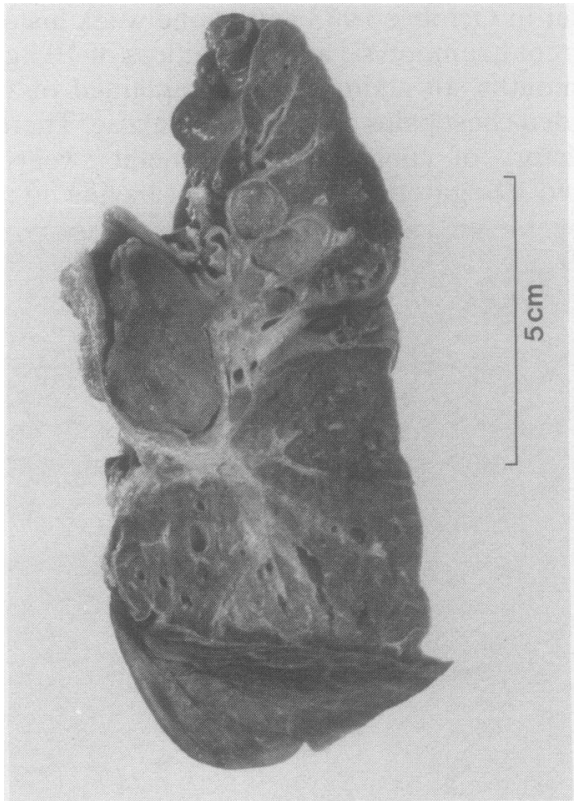

Fig 5 Case 3: Collapsed left lung with considerable bronchiectasis due to the presence of a whitish grey polypoid tumour arising in and completely obstructing the main bronchus. Tongues of tumour tissue are extending into the lumina of the adjacent segmental bronchi of the upper lobe. 
possible was removed with biopsy forceps until the bronchial lumen was almost cleared. Histological examination of the biopsy material showed features of a benign leiomyoma.

After bronchoscopy the patient began to expectorate pus. He continued to be febrile and his left lung failed to re-expand. Repeat bronchoscopy three weeks later showed further tumour, which appeared to be arising from the posterior wall of the left main bronchus close to the upper lobe orifice. Histological examination again showed fragments of a leiomyoma. Resection of the collapsed lung was decided on since the bronchus could not be cleared owing to the extent of the tumour.

At thoracotomy in September 1956 the left lung was completely atelectatic and adherent to the chest wall. A large mass was palpable at the hilum of the left upper lobe. There was inflammatory enlargement of the hilar lymph nodes. A left pneumonectomy was carried out (fig 5). Histological examination showed a benign leiomyoma.

The patient made an uneventful recovery, has remained entirely symptom free, and is now a 33 year old lecturer whose hobbies include cross country running and football.

\section{Discussion}

\section{INCIDENCE}

Benign tumours of the tracheobronchial tree comprise $4 \%$ of all surgically excised tumours. Leiomyomas are extremely rare and only 65 cases have so far been reported..$^{1-13}$ In a review of 128 benign tumours excised at the North Middlesex Hospital during 30 years $\mathrm{Hurt}^{8}$ found only two leiomyomas (one was of bronchial origin and the other was in the lung parenchyma); this parallels the experience of Arrigoni et $a^{\varphi}$ at the Mayo Clinic, where only two leiomyomas (one bronchial and the other parenchymal) were encountered among 130 benign tumours seen over a period of 10 years. Peleg and Pauzner ${ }^{10}$ in Israel reviewed 34 benign tumours and added one to the existing published cases. These reviews indicate that leiomyoma accounts for less than $2 \%$ of benign tumours of the lower respiratory tract. The trachea itself is the least common site, for only 10 such cases have been reported Gilbert $e t a^{11}$ found only two leiomyomas in their review of 256 benign tumours of the trachea.

\section{AGE AND SEX}

Leiomyomas are predominantly tumours of the young and the middle aged, with a mean age of 35 years for bronchial and lung parenchymal lesions ${ }^{2}$ and 40.6 years for tracheal lesions. ${ }^{5-7}$ The youngest patient with a bronchial leiomyoma was the 5 year old child reported here, and the youngest patient reported so far with a tracheal leiomyoma was a 3 month old infant. ${ }^{6}$ One third of patients present before the age of 20 years. $^{2}$ The oldest patient reported was $67 . .^{5}$

Leiomyomas of lung parenchyma occur twice as often in females as males, ${ }^{2}$ whereas leiomyomas of the trachea have been reported more often in males. ${ }^{6}$ Endobronchial lesions show no sex predilection. $^{4}$

\section{SITE AND PRESENTATION}

These tumours are more often encountered in the distal part of the tracheobronchial tree than proximally (table 1). Only three of the 51 cases reviewed by Orlowski et $a^{2}{ }^{2}$ arose from the main stem bronchi. The commonest segment of the trachea to be affected is the lower third and the usual site of origin is the posterior membranous portion. ${ }^{56}$

Over a third of leiomyomas are asymptomatic and are incidental findings on routine chest radiographs. ${ }^{3}$ This reflects the prevalence of peripheral and parenchymal lesions for these are asymptomatic in over $90 \%$ of cases. ${ }^{2}$ The presentation depends on the location of the tumour, its size, and changes in the lung distal to the lesion.

Patients with bronchial lesions all have symptoms due to partial or complete obstruction of the affected bronchus. These include wheeze, cough, chest pain, and fever, as a result of atelectasis, consolidation, collapse, or bronchiectasis. Haemoptysis is common and may reflect lung damage or ulceration of the surface of the lesion. With the risk of recurrent pneumonia and irreversible collapse of the lung, together with bronchiectasis and ultimate lung destruction, as in case 3 , there is a high premium on correct early diagnosis. Shahian and McEnany ${ }^{4}$ reported a case of pneumothorax secondary to air trapping beyond an endobronchial leiomyoma.

Intermittent or constant dyspnoea and wheezing are the most common symptoms of tracheal leiomyoma and have been erroneously ascribed to bronchial asthma. The duration of asthma like symptoms before correct diagnosis has been as long as nine years.' Wheezing due to an obstructing tracheal tumour must be distinguished from bron-

Table 1 Reported cases of leiomyoma of the lower respiratory tract $^{* 1-13}$

\begin{tabular}{lll}
\hline Site & $\begin{array}{l}\text { No of } \\
\text { cases }\end{array}$ & $\%$ \\
\hline Trachea & 12 & 16 \\
Bronchus & 22 & 33 \\
Parenchyma & 34 & 51 \\
Total & 68 & \\
\hline
\end{tabular}

*Including the three cases reported in this paper. 
chial asthma in any patient irrespective of age. Leiomyomas may produce paroxysmal attacks of wheezing, which are precipitated or relieved by a change in position, and there is no response to bronchodilator treatment. Other features include haemoptysis, which is associated with asthmatic wheezing, and orthopnoea or paroxysmal noctural dyspnoea in the absence of significant cardiovascular disease. The characteristic appearances of the flow volume loop of a patient with an obstructive lesion in the proximal bronchial tree distinguish an organic obstructive lesion from bronchial asthma.

\section{INVESTIGATION}

Most leiomyomas can be detected on plain chest radiographs but there are no pathognomonic features. Parenchymal tumours usually appear as rounded shadows but they cannot be distinguished clearly from other benign, malignant, or metastatic lesions. Most authors stress that the presence of calcification within rounded shadows on a chest radiograph argues against malignancy, although this is not a very reliable feature. In the two patients with leiomyomas reported by Crastnopol and Franklin' and Sweet $^{12}$ calcification was described. No calcification was seen in any of our cases.

Radiological features include the sequelae of bronchial obstruction. The tumour may be obscured by the features of atelectasis or pneumonitis. Hyperlucency due to air trapping and even pneumothorax have been reported. Tracheal leiomyomas may be seen on plain radiographs of the thoracic inlet, especially in the lateral view. ${ }^{13}$

Tomography is an excellent method of showing tracheal lesions and is useful for delineating leiomyomas in the rest of the bronchial tree. The place of computed tomography for evaluating these tumours has yet to be explored. The absolute diagnosis of leiomyomas of the lower respiratory tract is by bronchoscopy, which gives direct visualisation, and biopsy material is sent for histological examination. Frozen section is required, in the absence of a preoperative tissue diagnosis, when an atypical tumour is found at thoractomy. This obviates unnecessary lung resection.

\section{PATHOLOGY}

Leiomyomas of the trachea and bronchi arise from the smooth muscles of the tracheal and bronchial wall and grow as polypoid tumours, usually with broad bases. Typically bronchial leiomyomas extend along the bronchial lumen for a considerable distance, as tongues of soft tissue..$^{14}$ While tracheal and bronchial leiomyomas are very cellular neoplasms, with minimal vascular or stromal fibrous component, parenchymal lung tumours tend to be of a
Table 2 Surgically excised leiomyomas of the lower respiratory tract $^{* 1-13}$

\begin{tabular}{lcc}
\hline $\begin{array}{l}\text { Extent of } \\
\text { resection }\end{array}$ & $\begin{array}{l}\text { No of } \\
\text { cases }\end{array}$ & $\%$ \\
\hline Pneumonectomy & 8 & $14 \%$ \\
Lobectomy & 29 & $51 \%$ \\
Segmentectomy & 12 & $21 \%$ \\
Open local excision & 2 & $4 \%$ \\
Bronchoscopic local & 3 & $5 \%$ \\
$\quad$ excision & 3 & $5 \%$ \\
\hline
\end{tabular}

*Including the three cases reported in this paper.

more fibrous consistency and macroscopically have a whorled appearance. Histological examination of parenchymal pulmonary leiomyomas show them to consist largely of smooth muscle fibres, although an appreciable fibrous and vascular component is usually present. These lesions may be difficult to differentiate from fibromas, neurofibromas, or neurilemomas with ordinary haematoxylin and eosin stains. Special stains or electron microscopic examination may be required to establish their smooth muscle origin. ${ }^{45}$ Pulmonary leiomyomas are thought to arise from the smooth muscle of the bronchi, bronchioles, or blood vessels. ${ }^{4}$

Of particular interest is the fact that the histological appearances of parenchymal leiomyoma closely mimic those of the uterine fibroid. On rare occasions pulmonary tumours with features of benign fibroleiomyomas were shown to be metastatic deposits from uterine "fibroids" of an apparently benign and well differentiated nature. ${ }^{14}$ In describing these cases, Steiner ${ }^{16}$ coined the term "metastasising fibroleiomyoma of the uterus." There is considerable controversy about whether the pulmonary nodules represent metastases from an extremely well differentiated leiomyosarcoma or multicentric benign leiomyomatous growth. ${ }^{17}$ It may be unjustifiable ${ }^{14}$ to diagnose an intrapulmonary fibroleiomyoma in the absence of a complete examination of the uterus to exclude a malignant fibroid, even if the pulmonary lesion is solitary and apparently well encapsulated, and has minimal cytological atypia. Such a possibility, however, was excluded in our case of parenchymal pulmonary leiomyoma, as the patient (case 1) was a man.

Recently Horstmann et $a^{18}$ reviewed 14 cases of multiple leiomyomatous hamartomas consisting of bundles of smooth muscle, collagen fibres, and glandular epithelial cells. In one case the pulmonary nodules regressed spontaneously during pregnancy and the postpartum period, an observation that suggested hormonal dependence. ${ }^{18}$

The absence of epithelial or other mesenchymal components in the three cases documented here is in favour of a true neoplastic lesion rather than a 
hamartomatous malformation. We agree with Hurt $^{8}$ that the term hamartoma has been abused and should be applied only to lesions showing clear evidence of an underlying developmental abnormality, either present at birth or manifested by excessive growth during normal tissue maturation.

\section{TREATMENT}

The treatment of leiomyomas is essentially conservative surgery. There has been no report of recurrence after limited excision. Nevertheless extensive lung resection may be required as a result of bronchiectasis or chronic infective changes distal to the tumour. Of the 57 bronchopulmonary cases so far reported, $29(51 \%)$ required lobectomy and a further eight (14\%) pneumonectomy (table 2).

Parenchymal lesions usually require a less radical procedure and about one third have been treated by segmental resection, although a proximal parenchymal lesion compressing the bronchial tree may still make lobectomy necessary as in the first of our cases. It has been suggested that in many reported cases of lobectomy or pneumonectomy a less radical operation such as segmental or sleeve resection might have been preferable had the histological diagnosis been known before completion of the surgical procedure. ${ }^{2}$

Other conservative procedures include bronchotomy and local resection, and bronchoplastic resection, which has been used successfully in bronchial adenomas. ${ }^{419}$ Hurt $^{8}$ describes closure of a defect in the bronchial wall with a Gebauer skin graft. Only two bronchial leiomyomas have so far been successfully removed bronchoscopically. ${ }^{4}$ We found that in our case 3 the bronchial tumour was too extensive for complete removal, and in any event irreversible pulmonary changes beyond the lesion necessitated lung resection.

Tracheal leiomyomas have been dealt with in three ways: firstly, by bronchoscopic removal; secondly, by excision of the tumour via a tracheotomy incision as in our second case; and, thirdly, by circumferential resection with primary end to end anastomosis. ${ }^{7}$ Since these lesions are benign and well circumscribed, the most conservative treatment appropriate to the lesion is recommended. A broad base of attachment to the membranous portion of the trachea is, however, typical and this precludes bronchoscopic resection in most cases.
We thank Dr JC Briggs for reading the manuscript and Mrs G Caseley for invaluable secretarial assistance.

\section{References}

1 Crastnopol P, Franklin WD. Fibroleiomyoma of the lung. Ann Surg 1957;145:128-32.

2 Orlowski TM, Stasiak K, Kolodziej J. Leiomyoma of the lung.J Thoracic Cardiovasc Surg 1978;76:257-61.

3 Taylor TL, Miller DR. Leiomyoma of the bronchus. $J$ Thoracic Cardiovas Surg 1969;57:284-8.

4 Shahian DM, McEnany MT. Complete endobronchial excision of leiomyoma of the bronchus. J Thorac Cardiovasc Surg 1979;77:87-91.

5 Sanders JS, Cornes VM. Leiomyoma of the trachea. Report of a case, with a note on the diagnosis of partial tracheal obstruction. $N$ Engl J Med 1961;264:277-9.

6 Foroughi E. Leiomyoma of the trachea. Dis Chest 1962;42:230-2.

7 Kitamura S, Maeda M, Kawashima Y, Masaoka A, Manabe $H$. Leiomyoma of the intrathoracic trachea. $J$ Thorac Cardiovasc Surg 1969;57:126-33.

8 Hurt R. Benign tumours of the bronchus and trachea: 1951-1981. Ann $R$ Coll Surg 1984;66:22-6.

9 Arrigoni MG, Woolner LB, Bernatz PE, Miller WE, Fontana RS. Benign tumors of the lung. A ten-year surgical experience. $J$ Thorac Cardiovas Surg 1970;60:589-99.

10 Peleg H, Pauzner Y. Benign tumors of the lung. Dis Chest 1965;47:179-86.

11 Gilbert JG, Mazzarella LA, Feit LJ. Primary tracheal tumours in infant and adult. Arch Otolaryngol 1954;58:1-9.

12 Sweet RS. Pulmonary leiomyoma. Am J Roentgenol 1969;107:823-6.

13 Groves LK, Middlestone WR, McCormack LJ. Leiomyoma of the trachea: report of a case. Cleveland Clinic Quarterly 1958;25:32-7.

14 Spencer H. Pathology of the lung. Vol 1. 3rd ed. Oxford: Pergamon Press, 1977:895-7.

15 Ghadially FN. Diagnostic electron microscopy of tumours. London: Butterworth, 1980:128-53.

16 Steiner P. Metastasizing fibroleiomyoma of uterus: report of case and review of literature. Am J Pathol 1939;15:89-110.

17 Rosai J. Ackerman's surgical pathology. Vol 1. 6th ed. St Louis: Mosby, 1981:283-4.

18 Horstmann JP, Pietra GG, Harman JA, Cole NG, Grinspan S. Spontaneous regression of pulmonary leiomyomas during pregnancy. Cancer 1977;39:31421.

19 Jensik RJ, Faber LP, Brown CM, Little CF. Bronchoplastic and conservative resection procedures for bronchial adenomas. J Thorac Cardiovasc Surg 1974;68:556-65. 